\title{
Experimental demonstration of mode structure in ultralong Raman fiber lasers
}

\author{
S. A. Babin \\ Institute of Automation and Electrometry, Novosibirsk 630090, Russia \\ V. Karalekas and P. Harper \\ Photonics Research Group, Aston University, Birmingham, B4 7ET, UK \\ E. V. Podivilov \\ Institute of Automation and Electrometry, Novosibirsk 630090, Russia
}

V. K. Mezentsev, J. D. Ania-Castañón, and S. K. Turitsyn

Photonics Research Group, Aston University, Birmingham, B4 7ET, UK

Received January 2, 2007; accepted January 21, 2007;

posted February 7, 2007 (Doc. ID 78613); published April 3, 2007

\begin{abstract}
We present the first experimental demonstration of a resolvable mode structure with spacing $c / 2 n L$ in the $\mathrm{RF}$ spectra of ultralong Raman fiber lasers. The longest ever demonstrated laser cavity $(L=84 \mathrm{~km}), \mathrm{RF}$ peaks of $\sim 100 \mathrm{~Hz}$ width and spacing $\sim 1 \mathrm{kHz}$ have been observed at low intracavity powers. The width of the peaks increases linearly with growing intracavity power and is almost independent of fiber length. (C) 2007 Optical Society of America

OCIS codes: $190.5650,140.3550$.
\end{abstract}

The Raman fiber laser (RFL) is an example of a photonic system relying on the fundamental nonlinear optical effect of stimulated Raman scattering. The stimulated Raman scattering effect in a RFL manifests itself by shifting the spectrum of electromagnetic radiation propagating through an optical fiber towards longer wavelengths. It is then possible to overcome the fiber loss at the shifted Stokes wavelength by providing Raman amplification with a relatively low pump power $(<1 \mathrm{~W})$. As was demonstrated in Ref. 1 , by creating a cavity at the Stokes wavelength, e.g., applying fiber Bragg gratings (FBGs) as cavity reflectors, a Raman laser might be implemented in a $\sim 1 \mathrm{~km}$ long fiber waveguide. Since the Raman gain spectrum is rather broad in conventional germanosilicate fibers and multiple-order Stokes shifts can be achieved, RFLs can be designed to operate at almost any wavelength in the near-IR region $(1.1-1.7 \mu \mathrm{m})$. Moreover, tunable as well as multiwavelength RFL operation has been proved to be feasible. ${ }^{2}$ These quite unique features, coupled with the compactness, practicality, and potential for high efficiency, make RFLs very attractive CW light sources for a variety of applications, such as optical coherence tomography, ${ }^{3}$ long-distance remote sensing ${ }^{4}$ and especially in telecommunication, as pump and signal sources for distributed amplified systems (see, e.g., Ref. 5 and references therein).

The use of noise-efficient distributed Raman amplification in optical communication links has become popular in recent years thanks to the availability of high-power laser pumps, in particular, multiwavelength RFLs providing broadband gain with excellent spectral flatness. This has, in turn, spawned a great deal of interest in research on efficient control and optimization of the signal power evolution within the fiber span (see, e.g., Refs. 5 and 6 and references therein, for an overview of the literature). Recently, an interesting realization of ultralong Raman laser architecture for quasi-lossless signal transmission in fiber communication links has been proposed and implemented. ${ }^{7-9}$ In such an ultralong laser, the combined forward- and backward-propagating power generated at the Stokes wavelength at $\sim 1455 \mathrm{~nm}$ inside the cavity of the RFL (formed in the transmission fiber itself) experiences reduced variations along the fiber span. Hence, it can be used as a secondary pump to provide a near constant Raman gain for optical signal propagating at $1550 \mathrm{~nm}$. Quasi-lossless transmission over $75 \mathrm{~km}$ within $36 \mathrm{~nm}$ bandwidth has been demonstrated in Ref. 9. Evidently, such a dramatic increase in the length of the RFL cavity leads to a new, interesting class of lasers with potentially different physical mechanisms underlying their operation, and the natural question arises as to which are the specific peculiarities and properties of such an unusually long RFL.

One of the important laser characteristics is the existence of longitudinal mode structure formed in the laser cavity. It has been shown recently ${ }^{10}$ that for a Raman laser with $L=0.37 \mathrm{~km}$ cavity length, a corresponding RFL mode structure with spacing as small as $\Delta=c / 2 n L \sim 0.3 \mathrm{MHz}$ ( $n$ is refractive index, $c$ is speed of light) is observable in its RF spectrum; however, the observed peaks are broadened significantly at $\sim 3 \mathrm{~W}$ power of the intracavity generated Stokes wave. An increase of the cavity length by two orders of magnitude reduces proportionally the mode spacing, which may be critical for resolving the mode structures. In this Letter we present the first experimental demonstration of mode structure in ultralong (up to $L=84 \mathrm{~km}$ ) RFLs. 
The basic design of the ultralong laser cavity studied in this work is schematically depicted in Fig. 1. Without loss of generality, we consider an ultralong Raman fiber laser (URFL) designed to provide distributed signal amplification in the telecommunication window at $1550 \mathrm{~nm}$. However, we stress that the concept of URFL presented here is very general and is not limited to the particular wavelengths considered in this Letter. The URFL system consists of two equal-power depolarized primary pumps centered at $1365 \mathrm{~nm}$ and launched from both ends of the standard single-mode fiber span. Two FBG reflectors centered at $1455 \mathrm{~nm}$ were used at either end of the fiber span. This results in the formation of a high- $Q$ cavity trapping the first Stokes counterpropagating waves between the two highly reflective FBGs (the reflection coefficient is 98\%). When the power of the primary pumps is above the required threshold for the Raman gain to overcome the fiber attenuation, the laser starts to generate radiation at the Stokes wavelength of $1455 \mathrm{~nm}$. Monitoring of the generated intracavity Stokes wave power $P$ at $1455 \mathrm{~nm}$ and the corresponding optical spectra has been implemented through the use of two 99:1 splitters located near the FBGs at both ends of the span. To examine the mode structure of the URFL cavity, the signals from the $1 \%$ splitter ends were also analyzed using a fast photodiode and an electrical spectrum analyzer with resolution of $\sim 400 \mathrm{~Hz}$ to obtain an RF spectrum in which intermode beating can be observed (see, e.g., Ref. 10). In this paper we study the mode structure in URFLs with single-mode fiber lengths of $L=6.6,22,44$, and $84 \mathrm{~km}$.

First, note that the optical spectrum of the generated Stokes wave extends over about $1 \mathrm{~nm}$. The generated $1455 \mathrm{~nm}$ power is lower and has a higher threshold value for longer fiber spans, due to increasing pump absorption, in accordance with the theoretical RFL model confirmed by experiments with the short fiber spans; see, e.g., Ref. 11.

The results of the $\mathrm{RF}$ beating spectra, measured slightly above the generation threshold, are presented in Fig. 2. It is clearly seen that the intermode beating peaks are resolvable for all the studied cavity lengths, and that the spectral spacing $\Delta$ between them follows the classical formula $\Delta=c / 2 n L$. For the longest cavity length $L=84 \mathrm{~km}$ (the current record for laser cavity length to the best of our knowledge), the corresponding mode spacing is as low as $\Delta$ $\approx 1.2 \mathrm{kHz}$, while the width of the RF peaks is narrowed to hundreds of $\mathrm{Hz}$. Furthermore, we have observed the broadening of the modal peaks for all the

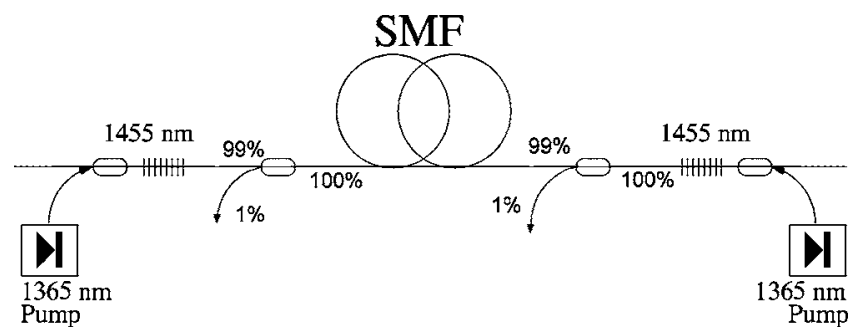

Fig. 1. Schematic depiction of the URFL.
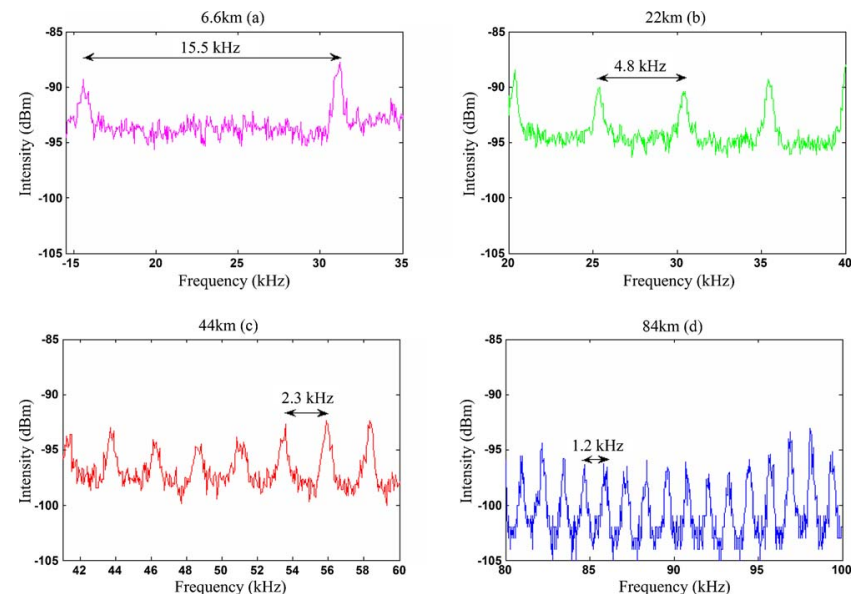

Fig. 2. (Color online) URFL RF spectra measured via the first splitter for different cavity lengths (a) $6.6 \mathrm{~km}$, (b) $22 \mathrm{~km}$, (c) $44 \mathrm{~km}$, and (d) $84 \mathrm{~km}$ at total intracavity power $P \approx 7 \mathrm{~mW}$ at $1455 \mathrm{~nm}$.

considered cavity lengths as the intracavity power is increased (see Fig. 3 for the particular case of $L$ $=6.6 \mathrm{~km}$ ). Figure 4 shows the measured spectral width (full width at half-maximum, i.e., spectral width at $-3 \mathrm{~dB}$ level) of the RF peaks versus the total intracavity power at $1455 \mathrm{~nm}$. It is clearly seen that the spectral width of the RF peaks depends on the intracavity power in a nearly linear manner and is almost independent of the fiber span length. The width values extrapolated to zero powers are as low as $\sim 250 \mathrm{~Hz}$, which nearly corresponds to the nameplate resolution of the electric spectrum analyzer used. One quite intriguing observation from the presented results is that propagation in such a long span does not drastically impact the relative phases of the modes, resulting in narrow RF peaks at low powers. It would be natural to expect that various effects such as thermal noise and fiber span fluctuations would lead to mode dephasing in so long laser cavities.

It is also expected that with the growing intracavity power nonlinear optical effects play an increasingly important role in the properties of laser modes. The observed broadening of the RF peaks with growing intracavity power is a clear manifestation of such nonlinear behavior. The role of the nonlinear effects in the spectral broadening of the emission radiation spectra in a relatively short RFL with a high- $Q$ cavity has been recently re-examined. ${ }^{12}$ It has been suggested $^{12}$ that with increasing intensity of laser radiation the main contribution to dephasing of the Stokes wave in the cavity comes from the effective "nonlinear attenuation" effect arising from the fourwave-mixing (FWM)-induced scattering of waves with different frequencies (corresponding to different cavity modes). This dephasing process is greatly enhanced in a long cavity by the multiple FWM processes caused by the large numbers of modes propagating simultaneously. In the case of URFL the mode number estimated as the ratio of the optical spectrum width and the mode spacing is really huge $\left(\sim 10^{8}\right)$. Nonlinear interactions of such a huge number of cavity modes lead to a linear dependence of the 

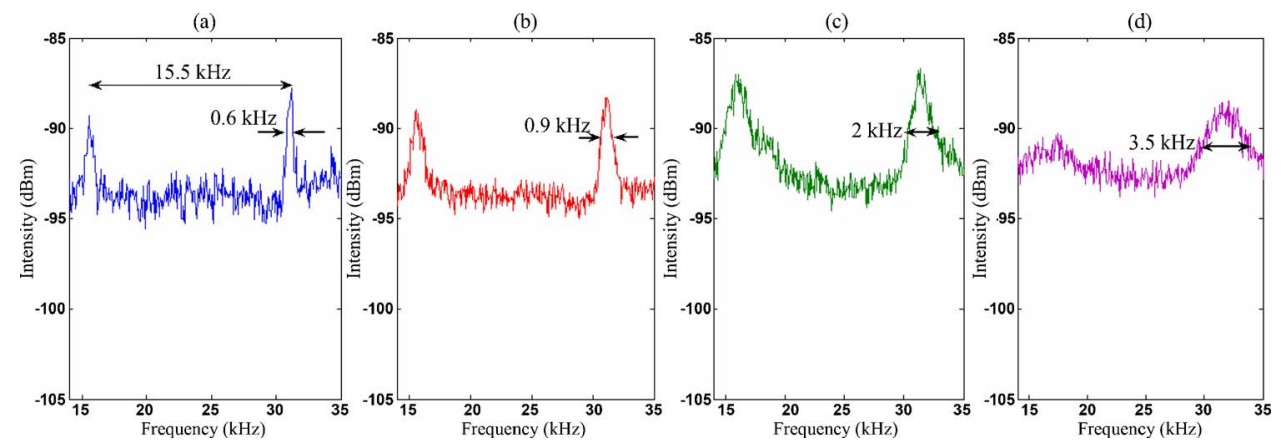

Fig. 3. (Color online) RF spectra measured via the first splitter for the RFL with cavity length $L=6.6 \mathrm{~km}$ at different total intracavity power at $1455 \mathrm{~nm}$ (a) $P \approx 7 \mathrm{~mW}$, (b) $61 \mathrm{~mW}$, (c) $136 \mathrm{~mW}$, (d) $265 \mathrm{~mW}$.

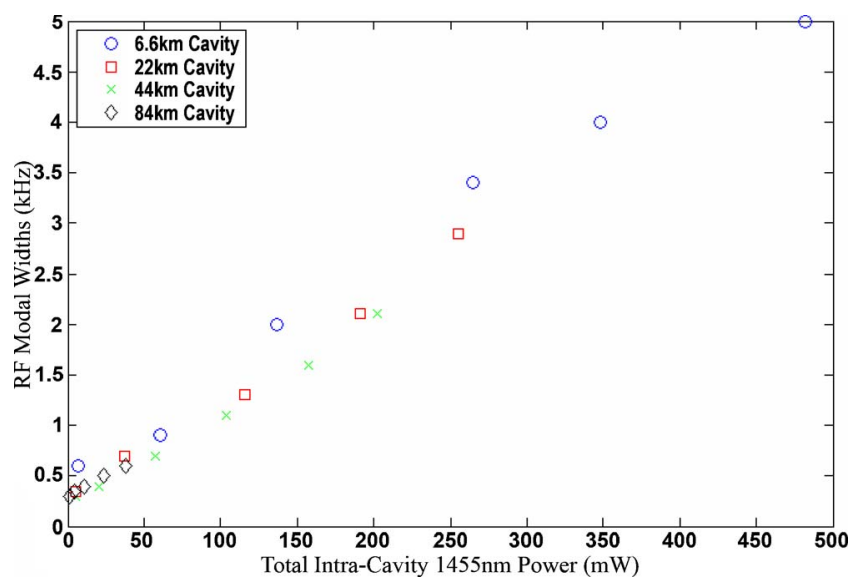

Fig. 4. (Color online) Spectral width of the RF peaks at $-3 \mathrm{~dB}$ level as a function of the total intracavity power at $1455 \mathrm{~nm}$.

RF peak width on the intracavity Stokes wave power. This is, to the best of our knowledge, the first experimental observation of this effect. The theoretical explanation is beyond the scope of this Letter and will be presented elsewhere.

In conclusion, we have experimentally demonstrated for the first time that the mode structure of the ultralong fiber laser can be resolved up to the record cavity length of $84 \mathrm{~km}$. The very narrow $\mathrm{RF}$ peaks reveal that the relative phase fluctuations between the neighboring modes remains very small, even propagating through such a long span. Thus, our results prove the feasibility of a new class of laser with an ultralong cavity. At the same time, significant dephasing occurs with an increase of the generated RFL power to the level of tens of $\mathrm{mW}$ inside the ultralong cavity due to their interaction via multiple FWM processes. These effects have an impact on the application of URFLs in telecommunications, i.e., for quasi-lossless transmission, since they determine the laser's ultimate relative intensity noise characteristics and should, therefore, be studied in further detail.
J. D. Ania-Castañón and S. K. Turitsyn acknowledge the support of the Engineering and Physical Sciences Research Council. S. A. Babin and E. V. Podivilov acknowledge the support through integration project N31 of the Siberian Branch of the Russian Academy of Sciences. V. Karalekas's e-mail address is karalekv@aston.ac.uk.

\section{References}

1. S. G. Grubb, T. Strasser, W. Y. Cheung, W. A. Reed, V. Mizrahi, T. Erdogan, P. J. Lemaire, A. M. Vengsarkar, and D. J. Digiovanni, in Optical Amplifiers and Their Applications, Vol. 18 of OSA Technical Digest Series (Optical Society of America, 1995), paper SaA4.S7.

2. M. D. Mermelstein, C. Headley, J.-C. Bouteiller, P. Steinvurzel, C. Horn, K. Feder, and B. J. Eggleton, IEEE Photon. Technol. Lett. 13, 1286 (2001).

3. P.-L. Hsiung, Y. Chen, T. H. Ko, J. G. Fujimoto, C. J. S. de Matos, S. V. Popov, J. R. Taylor, and V. P. Gapontsev, Opt. Express 12, 5287 (2005).

4. Y.-G. Han, T. V. A. Tran, S.-H. Kim, and S. B. Lee, Opt. Lett. 30, 1114 (2005).

5. C. Headley and G. P. Agrawal, Raman Amplification in Fiber Optical Communication Systems (Academic, 2004).

6. D. A. Chestnut, C. J. S. de Matos, P. C. Reeves-Hall, and J. R. Taylor, Opt. Lett. 27, 1708 (2002).

7. J. D. Ania-Castañón, Opt. Express 12, 4372 (2004).

8. J. D. Ania-Castañón, T. J. Ellingham, R. Ibbotson, X. Chen, L. Zhang, and S. K. Turitsyn, Phys. Rev. Lett. 96, 023902 (2006).

9. T. J. Ellingham, J. D. Ania-Castañón, R. Ibbotson, X. Chen, L. Zhang, and S. K. Turitsyn, IEEE Photon. Technol. Lett. 18, 268 (2006).

10. S. A. Babin, D. V. Churkin, A. A. Fotiadi, S. I. Kablukov, O. I. Medvedkov, and E. V. Podivilov, IEEE Photon. Technol. Lett. 17, 2553 (2005).

11. S. A. Babin, D. V. Churkin, and E. V. Podivilov, Opt. Commun. 226, 329 (2003).

12. S. A. Babin, D. V. Churkin, A. E. Ismagulov, S. I. Kablukov, and E. V. Podivilov, Opt. Lett. 31, 3007 (2006). 